\title{
CONTINUATION AND UNIQUENESS FOR THREE DIMENSIONAL MIRZOV'S SYSTEMS
}

\author{
JOZEF KISEL'ÁK
}

\begin{abstract}
We discuss continuation and uniqueness of solutions to the Cauchy problem for a three dimensional Mirzov's system. Moreover, two specific cases are partially analyzed .
\end{abstract}

Mathematics Subject Classification 2000: 34A12

General Terms: Theory

Additional Key Words and Phrases: Global solution, Mirzov's differential system, uniqueness

\section{INTRODUCTION}

We are concerned with the third-order Mirzov's differential system

$$
\begin{aligned}
& y_{0}^{\prime}=r_{1}(t) \phi_{\alpha_{1}}^{-1}\left[y_{1}\right], \\
& y_{1}^{\prime}=r_{2}(t) \phi_{\alpha_{2}}^{-1}\left[y_{2}\right], \\
& y_{2}^{\prime}=q(t) \phi_{\beta}\left[y_{0}\right] .
\end{aligned}
$$

where $\beta=\alpha_{1} \alpha_{2}, \alpha_{1}>0, \alpha_{2}>0, \quad q, r_{i} \in C(I), I=\langle a, \infty), i=1,2$, and $\phi_{\alpha}[x]:=|x|^{\alpha-1} x, \alpha>0$, known as signed power function. Moreover we assume that $r_{1}, r_{2}$ are positive functions on half-line $I$. One of the pioneering works in the qualitative theory of such system is the paper of Mirzov [Mirzov 1976], who has studied such systems intensively. In that paper, the analogue of two-dimensional first order differential system is considered. System $(E)$ can be rewritten as differential equation of the third-order. By the analogy of second-order it can be named half-linear, which expresses the fact, that a solution space is homogeneous but not additive. This kind of quasilinear systems are really challenging. Authors in [Ollagnier et al. 1995] use Bezout's theorem to prove that the so-called Jouanolou system

$$
x^{\prime}=y^{n}, y^{\prime}=z^{n}, z^{\prime}=x^{n}, \quad 2 \leq n \in \mathbb{N},
$$

does not admit any Darboux polynomials. Hence, it does not have a rational or algebraic first integral. Moreover the so-called Emden-Fowler equation (less general than our system) of any index is well-known to be rather sparing of integrable cases. Motivation could be specific models from three-dimensional urn process theory called cyclic chambers (or generalized Ehrenfest urns), where so-called Poincaré cycles are to be calculated. To get detailed information, reader should see [Flajolet et al. 2006].

The paper is organized as following. First we discuss the global existence of a 
solution of $(E)$ subject to the initial conditions. The proof is based on the classical results, like the Peano theorem and the Gronwall inequality. It is done for the case $\beta=\alpha_{1} \alpha_{2}$ but as it is shown below $\beta \leq \alpha_{1} \alpha_{2}$ inequality is sufficient. We also offer an example which contradicts the remaining case. In the second section we analyze more subtle problem, the uniqueness of the solutions. We emphasize usage of generalized Riccati transformation (6), which proves to be useful. A Cauchy problem (the case $\beta=\alpha_{1} \alpha_{2}$ ) has for all initial values a unique solution and the only restriction is condition $q(a) \neq 0$. Lack of Lipschitz condition for right-hand side brings for general $\beta$ (be more specific for $\beta \leq \alpha_{1} \alpha_{2}$ ) difficulties which should be overcome by additional assumption on q. But this exceeds our interest and reader can see [Erbe and Liang 1991], where continuation and uniqueness of solutions of the two-dimensional generalized Emden-Fowler system is studied. It seems that solutions of Mirzov's systems partially form a boundary between solutions, which are not continuable and solutions, which does not posses uniqueness. This is captured in main theorems 2.5 and 3.9 given in the article.

\section{EXISTENCE AND CONTINUATION OF THE IVP}

The question of continuation problem of such systems started probably when in [Coffman and Ullrich 1967] for the prototype of 2nd-order quasi-linear equation, $x^{\prime \prime}+c(t)|x|^{\gamma} \operatorname{sgn} x=0$, it was solved. Since then it was proved for several generalizations or related problems, we refer to [Kitano and Kusano 1995], where the problem with oscillating coefficient is studied. Surprisingly, as far as we know, there is no work on the third-order system or equation (Mrizov's system). Consider now the IVP

$$
(E), y_{0}\left(t_{0}\right)=A, y_{1}\left(t_{0}\right)=B, y_{2}\left(t_{0}\right)=C .
$$

From the right side of $(E)$ we know that IVP (2) has a local solution for any real values $A, B, C$. It follows from the Peano theorem. We now show, that such solution can be extended over the entire interval $[a, \infty)$ uder the condition $\beta \leq \alpha_{1} \alpha_{2}$.

LEMmA 2.1. The IVP (2) with $t_{0}=a$ and $\beta=\alpha_{1} \alpha_{2}$ has at least one solution defined on the whole interval $[a, \infty)$.

Proof. Choose $b \in[a, \infty)$ and integrate $(E)$ over the interval $[a, b]$. Hence, we obtain

$$
\begin{aligned}
& y_{0}(t)=y_{0}(a)+\int_{a}^{t} r_{1}(s) \phi_{\alpha_{1}}^{-1}\left[y_{1}(s)\right] \mathrm{d} s, \\
& y_{1}(t)=y_{1}(a)+\int_{a}^{t} r_{2}(s) \phi_{\alpha_{2}}^{-1}\left[y_{2}(s)\right] \mathrm{d} s, \\
& y_{2}(t)=y_{2}(a)+\int_{a}^{t} q(s) \phi_{\beta}\left[y_{0}(s)\right] \mathrm{d} s,
\end{aligned}
$$

for $t \in[a, b]$. Using the well-known Hölder's inequality, we obtain

$$
\left|y_{0}(t)-y_{0}(a)\right|^{\beta+\alpha_{1}+1} \leq\left(\int_{a}^{t} r_{1}(s)^{\beta_{0}}\right)^{\beta+\alpha_{1}} \int_{a}^{t}\left|y_{1}(s)\right|^{\frac{1}{\alpha_{1}}+\alpha_{2}+1} \mathrm{~d} s,
$$




$$
\begin{aligned}
\left|y_{1}(t)-y_{1}(a)\right|^{\frac{1}{\alpha_{1}}+\alpha_{2}+1} & \leq\left(\int_{a}^{t} r_{2}(s)^{\beta_{1}}\right)^{\alpha_{2}+1} \int_{a}^{t}\left|y_{2}(s)\right|^{\frac{1}{\beta}+\frac{1}{\alpha_{2}}+1} \mathrm{~d} s, \\
\left|y_{2}(t)-y_{2}(a)\right|^{\frac{1}{\beta}+\frac{1}{\alpha_{2}}+1} & \leq\left(\int_{a}^{t}|q(s)|^{\beta_{2}}\right)^{\frac{1+\alpha_{1}}{\beta}} \int_{a}^{t}\left|y_{0}(s)\right|^{\beta+\alpha_{1}+1} \mathrm{~d} s,
\end{aligned}
$$

where $\beta_{0}=1+\frac{1}{\beta+\alpha_{1}}, \beta_{1}=1+\frac{\alpha_{1}}{1+\beta}, \beta_{2}=1+\frac{\beta}{1+\alpha_{1}}$. Denote by

$$
M=2^{\beta+\alpha_{1}}\left|y_{0}(a)\right|^{\beta+\alpha_{1}+1}+2^{\frac{1}{\alpha_{1}}+\alpha_{2}}\left|y_{1}(a)\right|^{\frac{1}{\alpha_{1}}+\alpha_{2}+1}+2^{\frac{1}{\beta}+\frac{1}{\alpha_{2}}}\left|y_{2}(a)\right|^{\frac{1}{\beta}+\frac{1}{\alpha_{2}}+1}
$$

and

$$
G(b)=\max _{t \in[a, b]}\left\{\begin{array}{c}
2^{\beta+\alpha_{1}}\left(\int_{a}^{t} r_{1}(s)^{\beta_{0}}\right)^{\beta+\alpha_{1}}, 2^{\frac{1}{\alpha_{1}}+\alpha_{2}}\left(\int_{a}^{t} r_{2}(s)^{\beta_{1}}\right)^{\alpha_{2}+1}, \\
2^{\frac{1}{\beta}+\frac{1}{\alpha_{2}}}\left(\int_{a}^{t}|q(s)|^{\beta_{2}}\right)^{\frac{1+\alpha_{1}}{\beta}}
\end{array}\right\} .
$$

Then, we have from the inequality

$$
\left|x_{1}+x_{2}\right|^{p} \leq 2^{p-1}\left(\left|x_{1}\right|^{p}+\left|x_{2}\right|^{p}\right), \quad x_{1}, x_{2} \in \mathbb{R}, p \geq 1,
$$

that for $p=\beta+\alpha_{1}+1, p=\frac{1}{\alpha_{1}}+\alpha_{2}+1$ and $p=\frac{1}{\beta}+\frac{1}{\alpha_{2}}+1$ respectively, holds

$$
\begin{gathered}
\left|y_{0}(t)\right|^{\beta+\alpha_{1}+1}+\left|y_{1}(t)\right|^{\frac{1}{\alpha_{1}}+\alpha_{2}+1}+\left|y_{2}(t)\right|^{\frac{1}{\beta}+\frac{1}{\alpha_{2}}+1} \leq \\
M+2^{\beta+\alpha_{1}}\left(\int_{a}^{t} r_{1}(s)^{\beta_{0}}\right)^{\beta+\alpha_{1}} \int_{a}^{t}\left|y_{1}(s)\right|^{\frac{1}{\alpha_{1}}+\alpha_{2}+1} \mathrm{~d} s+ \\
+2^{\frac{1}{\alpha_{1}}+\alpha_{2}}\left(\int_{a}^{t} r_{2}(s)^{\beta_{1}}\right)^{\alpha_{2}+1} \int_{a}^{t}\left|y_{2}(s)\right|^{\frac{1}{\beta}+\frac{1}{\alpha_{2}}+1} \mathrm{~d} s+ \\
2^{\frac{1}{\beta}+\frac{1}{\alpha_{2}}}\left(\int_{a}^{t} q(s)^{\beta_{2}}\right)^{\frac{1+\alpha_{1}}{\beta}} \int_{a}^{t}\left|y_{0}(s)\right|^{\beta+\alpha_{1}+1} \mathrm{~d} s \leq \\
\leq M+G(b) \int_{a}^{t}\left(\left|y_{0}(s)\right|^{\beta+\alpha_{1}+1}+\left|y_{1}(s)\right|^{\frac{1}{\alpha_{1}}+\alpha_{2}+1}+\left|y_{2}(s)\right|^{\frac{1}{\beta}+\frac{1}{\alpha_{2}}+1}\right) \mathrm{d} s
\end{gathered}
$$

for $t \in[a, b]$. But using the Gronwall inequality, we finally have

$$
\left|y_{0}(t)\right|^{\beta+\alpha_{1}+1}+\left|y_{1}(t)\right|^{\frac{1}{\alpha_{1}}+\alpha_{2}+1}+\left|y_{2}(t)\right|^{\frac{1}{\beta}+\frac{1}{\alpha_{2}}+1} \leq M \mathrm{e}^{G(b)(t-a)}
$$

for $t \in[a, b]$. Thus the solution can be extended over the whole interval $[a, b]$ and since $b$ is arbitrary we are done.

One would expect, alike in the theory of lower-order differential equations, it can not be true in general (for arbitrary $\alpha_{1}, \alpha_{2}, \beta$ ) that solution is extendable over whole half-line. We give simple counterexamples for the case $\beta>\alpha_{1} \alpha_{2}$.

Example 2.2. Consider the IVP

$$
\begin{array}{ll}
x_{0}^{\prime}=x_{1}^{\frac{1}{3}}, & x_{0}(0)=3 \\
x_{1}^{\prime}=x_{2}, & x_{1}(0)=b^{3} \\
x_{2}^{\prime}=k\left|x_{0}\right|^{2+\frac{15}{b}} x_{0}, & x_{2}(0)=b^{3}(b+3)
\end{array}
$$


where, $b>0, \beta=3+\frac{15}{b}$ and $k=3^{-\beta} b^{3}\left(b^{2}+7 b+12\right)$. It is clear that $\alpha_{1} \alpha_{2}=3<\beta$ and that

$$
\left\{3(1-t)^{-\frac{b}{3}}, \quad b^{3}(1-t)^{-b-3}, \quad b^{3}(b+3)(1-t)^{-b-4}\right\}
$$

solves problem (4). But one can see that the solution blows up at $t=1$.

Example 2.3. The IVP with nonconstant coeficient $q$

$$
\begin{aligned}
x_{0}^{\prime} & =x_{1}, & x_{0}(0) & =1 \\
x_{1}^{\prime} & =\left|x_{2}\right|^{\frac{a-4}{6}} x_{2}, & x_{1}(0) & =a \\
x_{2}^{\prime} & =q(t) x_{0}^{7}, & x_{2}(0) & =(a(a+3))^{\frac{6}{a+2}}
\end{aligned}
$$

where $\alpha_{2}=\frac{6}{a+2}, a>0$ and $q(t)=\alpha a^{\alpha} \mathrm{e}^{\alpha t}\left(a^{2} \mathrm{e}^{2 t}+6 a \mathrm{e}^{t}+2 e^{t}+4\right)\left(a e^{t}+2\right)^{\alpha-1}$. Again $\beta=7>\alpha_{1} \alpha_{2}$ and (5) possesses solution

$$
\left\{\frac{1}{\left(2-\mathrm{e}^{t}\right)^{a}}, \frac{a \mathrm{e}^{t}}{\left(2-\mathrm{e}^{t}\right)^{a+1}}, \quad\left(\frac{a \mathrm{e}^{t}\left(a \mathrm{e}^{t}+2\right)}{\left(2-\mathrm{e}^{t}\right)^{a+2}}\right)^{\frac{6}{a+2}}\right\},
$$

which blows up at $t=\ln 2$.

Remark 2.4. One has to notice important fact. Let have following type of differential system: $\alpha_{1} \alpha_{2}>\beta$. Then inequalities

$$
\alpha_{2}>\frac{\beta}{\alpha_{1}}, \alpha_{1}>\frac{\beta}{\alpha_{2}}, \frac{1}{\alpha_{1} \alpha_{2}}<\frac{1}{\beta},
$$

give us upper bound for terms in Hölder inequalities in the proof of lemma 2.1 and thus blow-up can not appear also in the case $\alpha_{1} \alpha_{2}>\beta$ and the following theorem summarizes achieved facts.

TheOrem 2.5. The IVP (2) with $t_{0}=a$ and $\alpha_{1} \alpha_{2} \geq \beta$ has at least one solution defined on the whole interval $[a, \infty)$.

\section{UNIQUENESS OF THE IVP}

Now we take into account uniqueness of the solution, which is a subtle problem also for second-order half-linear differential equation (or equivalent system respectively) as is pointed out in [Reichel and Walter 1997]. From the relation $\beta=\alpha_{1} \alpha_{2}$ we know that

$$
\begin{aligned}
& \text { if } \alpha_{1} \leq 1, \alpha_{2} \leq 1 \text { then } \beta \leq 1 \\
& \text { if } \alpha_{1} \geq 1, \alpha_{2} \geq 1 \text { then } \beta \geq 1 \\
& \text { if } \beta<1 \text { then at least one of } \alpha_{i}<1, i=1,2 .
\end{aligned}
$$

From the Picard theorem it is ensured that the local solution of (2) is unique

for any $A \in \mathbb{R} \backslash\{0\}, B \in \mathbb{R}, C \in \mathbb{R}$ if $\beta<1, \alpha_{1} \leq 1, \alpha_{2} \leq 1$, for any $A \in \mathbb{R}, B \in \mathbb{R}, C \in \mathbb{R} \backslash\{0\} \quad$ if $\beta \geq 1, \alpha_{1} \leq 1, \alpha_{2}>1$, for any $A \in \mathbb{R}, B \in \mathbb{R} \backslash\{0\}, C \in \mathbb{R}$ if $\beta \geq 1, \alpha_{1}>1, \alpha_{2} \leq 1$, for any $A \in \mathbb{R}, B \in \mathbb{R} \backslash\{0\}, C \in \mathbb{R} \backslash\{0\} \quad$ if $\beta>1, \alpha_{1}>1, \alpha_{2}>1$, for any $A \in \mathbb{R} \backslash\{0\}, B \in \mathbb{R}, C \in \mathbb{R} \backslash\{0\} \quad$ if $\beta<1, \alpha_{1} \leq 1, \alpha_{2}>1$ and for any $A \in \mathbb{R} \backslash\{0\}, B \in \mathbb{R} \backslash\{0\}, C \in \mathbb{R}$ if $\beta<1, \alpha_{1}>1, \alpha_{2} \leq 1$. 
We differ two approaches. In the second and third case we use very usefull generalized Riccati transformation. For the rest of the cases we change problem into integral equations.

Thus we start with introducing generalized Riccati transformation. It is very useful as no additional restrictions about differentiability of coefficients are needed and the order of the system is reduced. If $y_{0}$ is a locally non-vanishing solution of the equation $(E)$ we make the following change of variable :

$$
u_{1}(t)=\frac{y_{1}(t)}{\phi_{\alpha_{1}}\left[y_{0}(t)\right]}, \quad u_{2}(t)=\frac{y_{2}(t)}{\phi_{\beta}\left[y_{0}(t)\right]}
$$

to obtain two dimensional nonlinear generalized Riccati system

$$
\begin{aligned}
& u_{1}^{\prime}=r_{2} \phi_{\alpha_{2}}^{-1}\left[u_{2}\right]-\alpha_{1} r_{1} u_{1} \phi_{\alpha_{1}}^{-1}\left[u_{1}\right], \\
& u_{2}^{\prime}=q-\beta r_{1} u_{2} \phi_{\alpha_{1}}^{-1}\left[u_{1}\right] .
\end{aligned}
$$

Lemma 3.1. Suppose that $A \neq 0, B \neq 0, C=0$ and $\alpha_{2}>1$ and $q(a) \neq 0$. Then the IVP (2) with $t_{0}=a$ has a unique solution in a right neighborhood of the point a.

Proof. Unfortunately Riccati system is not Lipchitzian. We know that $u_{1}(a) \neq$ 0 and $u_{2}(a)=0$. Let be a solution $\left(u_{1}, u_{2}\right)$ of the Cauchy problem such that it is nonzero on $(a, a+\delta]$. Impose now functions

$$
\begin{aligned}
& v_{1}(t)=\frac{u_{1}(t)-u_{1}(a)}{t-a}, \\
& v_{2}(t)=\frac{u_{2}(t)}{t-a},
\end{aligned}
$$

which are continuous on $[a, a+\delta]$ and notice that $v_{2}(a)=q(a)$. We have for two functions $\left(v_{1}, v_{2}\right),\left(\hat{v}_{1}, \hat{v}_{2}\right)$ local relationships $v_{i}(t)-\hat{v}_{i}(t)=\frac{u_{i}(t)-\hat{u}_{i}(t)}{t-a}, \quad i=1,2$. Therefore, from the fact that $s-a \leq t-a$ for $t \in[a, a+\delta]$ we have

$$
\begin{gathered}
\left|v_{1}(t)-\hat{v}_{1}(t)\right| \leq \int_{a}^{t}(s-a)^{\frac{1}{\alpha_{2}}-1} r_{2}(s)\left|\phi_{\alpha_{2}}^{-1}\left[v_{2}(s)\right]-\phi_{\alpha_{2}}^{-1}\left[\hat{v}_{2}(s)\right]\right| \mathrm{d} s+ \\
+\alpha_{1} \int_{a}^{t}(s-a)^{\frac{1}{\alpha_{1}}} r_{1}(s)|| v_{1}(s)+\left.\frac{u_{1}(a)}{s-a}\right|^{1+\frac{1}{\alpha_{1}}}-\left|\hat{v}_{1}(s)+\frac{u_{1}(a)}{s-a}\right|^{1+\frac{1}{\alpha_{1}}} \mid \mathrm{d} s,
\end{gathered}
$$

and

$$
\begin{gathered}
\left|v_{2}(t)-\hat{v}_{2}(t)\right| \leq \\
\beta \int_{a}^{t}(s-a)^{\frac{1}{\alpha_{1}}} r_{1}(s)\left|v_{2}(s) \phi_{\alpha_{1}}^{-1}\left[v_{1}(s)+\frac{u_{1}(a)}{s-a}\right]-\hat{v}_{2}(s) \phi_{\alpha_{1}}^{-1}\left[\hat{v}_{1}(s)+\frac{u_{1}(a)}{s-a}\right]\right| \mathrm{d} s .
\end{gathered}
$$

Now we know from the mean value theorem than there exists

$$
\xi_{1}(s)=\left(1-\theta_{1}\right) v_{1}(s)+\theta_{1} \hat{v}_{1}(s)+\frac{u_{1}(a)}{s-a}, \quad \theta_{1} \in(0,1)
$$


s.t.

$$
|| v_{1}(s)+\left.\frac{u_{1}(a)}{s-a}\right|^{1+\frac{1}{\alpha_{1}}}-\left.\left|\hat{v}_{1}(s)+\frac{u_{1}(a)}{s-a}\right|^{1+\frac{1}{\alpha_{1}}}\left|\leq \frac{\alpha_{1}+1}{\alpha_{1}}\right| \xi_{1}(s)\right|^{\frac{1}{\alpha_{1}}}\left|v_{1}(s)-\hat{v}_{1}(s)\right|,
$$

where $\left|\xi_{1}(s)\right|^{\frac{1}{\alpha_{1}}}(s-a)^{\frac{1}{\alpha_{1}}}$ is clearly integrable on $[a, a+\delta]$. In similar fashion we have

$$
\left|\phi_{\alpha_{2}}^{-1}\left[v_{2}(s)\right]-\phi_{\alpha_{2}}^{-1}\left[\hat{v}_{2}(s)\right]\right| \leq \frac{\left|\xi_{2}(s)\right|^{\frac{1}{\alpha_{2}}-1}}{\alpha_{2}}\left|v_{2}(s)-\hat{v}_{2}(s)\right|
$$

where again

$$
\xi_{2}(s)=\left(1-\theta_{2}\right) v_{2}(s)+\theta_{2} \hat{v}_{2}(s), \quad \theta_{2} \in(0,1)
$$

and $\left|\xi_{2}(s)\right|^{\frac{1}{\alpha_{2}}-1}$ is integrable on $[a, a+\delta]$, because we know that $\xi_{2}(s) \neq 0$ for any $s \in[a, a+\delta]$ (in this case we have $\alpha_{2}>1$ ) since $q(a) \neq 0$. Moreover, using the mean value theorem for function of two variables we can get estimate

$$
\begin{gathered}
\left|v_{2}(s) \phi_{\alpha_{1}}^{-1}\left[v_{1}(s)+\frac{u_{1}(a)}{s-a}\right]-\hat{v}_{2}(s) \phi_{\alpha_{1}}^{-1}\left[\hat{v}_{1}(s)+\frac{u_{1}(a)}{s-a}\right]\right| \leq\left|\bar{\xi}_{1}(s)\right|^{\frac{1}{\alpha_{1}}}\left|v_{2}(s)-\hat{v}_{2}(s)\right|+ \\
+\frac{\left|\bar{\xi}_{1}(s)\right|^{\frac{1}{\alpha_{1}}-1}}{\alpha_{1}}\left|\bar{\xi}_{2}(s)\right|\left|v_{1}(s)-\hat{v}_{1}(s)\right|,
\end{gathered}
$$

where

$$
\bar{\xi}_{1}(s)=(1-\theta) v_{1}(s)+\theta \hat{v}_{1}(s)+\frac{u_{1}(a)}{s-a}, \quad \bar{\xi}_{2}(s)=(1-\theta) v_{2}(s)+\theta \hat{v}_{2}(s), \quad \theta \in(0,1),
$$

and clearly $\left|\bar{\xi}_{1}(s)\right|^{\frac{1}{\alpha_{1}}-1}\left|\bar{\xi}_{2}(s)\right|(s-a)^{\frac{1}{\alpha_{1}}},\left|\bar{\xi}_{1}(s)\right|^{\frac{1}{\alpha_{1}}}(s-a)^{\frac{1}{\alpha_{1}}}$ are integrable on $[a, a+\delta]$. Together we have inequality

$$
\left|v_{1}(t)-\hat{v}_{1}(t)\right|+\left|v_{2}(t)-\hat{v}_{2}(t)\right| \leq \int_{a}^{t} H(s, \delta)\left(\left|v_{1}(t)-\hat{v}_{1}(t)\right|+\left|v_{2}(t)-\hat{v}_{2}(t)\right|\right) \mathrm{d} s,
$$

where

$$
\begin{gathered}
H(s, \delta)=\max _{s \in[a, a+\delta]}\left\{\left(\alpha_{1}+1\right) r_{1}(s)\left|\xi_{1}(s)\right|^{\frac{1}{\alpha_{1}}}(s-a)^{\frac{1}{\alpha_{1}}}, \frac{r_{2}(s)}{\alpha_{2}}\left|\xi_{2}(s)\right|^{\frac{1}{\alpha_{2}}-1}(s-a)^{\frac{1}{\alpha_{2}}-1},\right. \\
\left., \beta R_{1}(s)(s-a)^{\frac{1}{\alpha_{1}}}\left|\bar{\xi}_{1}(s)\right|^{\frac{1}{\alpha_{1}}}, \alpha_{2}(s-a)^{\frac{1}{\alpha_{1}}}\left|\bar{\xi}_{2}\right|\left|\bar{\xi}_{1}(s)\right|^{\frac{1}{\alpha_{2}}-1}\right\} .
\end{gathered}
$$

Using Gronwall inequality it follows that $v_{1}=\hat{v}_{1}, v_{2}=\hat{v}_{2}$. This completes the proof.

The proof of the following lemma is similar and we omit it.

Lemma 3.2. Suppose that $A \neq 0, B=0, C \neq 0$ and $\alpha_{1}>1$. Then the IVP (2) with $t_{0}=a$ has a unique solution in a right neighborhood of the point $a$.

For the rest cases we need to rewrite our system as differential equation of the third order

$$
\left(\phi_{\alpha_{2}}\left[\frac{1}{r_{2}(t)}\left(\phi_{\alpha_{1}}\left[\frac{y_{0}^{\prime}}{r_{1}(t)}\right]\right)^{\prime}\right]\right)^{\prime}=q(t) \phi_{\beta}\left[y_{0}\right]
$$


The main idea of the proofs of following lemmas is to redefine a special function to be continuous in order to obtain a correct estimate. Therefore we prove next lemma in detial. The proofs of remaining lemmas are made in similar manner and we omit details and emphasize the main idea.

Lemma 3.3. Suppose that $A=0, B \neq 0, C \neq 0$ and $\beta<1$. Then the IVP (2) with $t_{0}=a$ has a unique solution in a right neighborhood of the point $a$.

Proof. Denote by $y_{01}$ and $y_{02}$ two local solutions of (2) with assumptions above. Now, let $t \in$ $\operatorname{dom}\left(y_{01}\right) \cap$

$\operatorname{dom}\left(y_{02}\right)$ and integrate (8) with $y=y_{0 i}$ twice from $a$ to $t$. If we denote as $I_{i}(t)=$ $\int_{a}^{t} q(s) \phi_{\beta}\left[y_{0 i}(s)\right] \mathrm{d} s$, we have

$$
y_{0 i}^{\prime}(t)=r_{1}(t) \phi_{\alpha_{1}}^{-1}\left[B+\int_{a}^{t} r_{2}(s) \phi_{\alpha_{2}}^{-1}\left[C+I_{i}(s)\right] \mathrm{d} s\right], i=1,2 .
$$

But this implies

$$
\begin{aligned}
y_{01}(t) & -y_{02}(t)=\int_{a}^{t} r_{1}(s) \phi_{\alpha_{1}}^{-1}\left[B+\int_{a}^{s} r_{2}(u) \phi_{\alpha_{2}}^{-1}\left[C+I_{1}(u)\right] \mathrm{d} u\right] \mathrm{d} s \\
& -\int_{a}^{t} r_{1}(s) \phi_{\alpha_{1}}^{-1}\left[B+\int_{a}^{s} r_{2}(u) \phi_{\alpha_{2}}^{-1}\left[C+I_{2}(u)\right] \mathrm{d} u\right] \mathrm{d} s .
\end{aligned}
$$

Further we use the mean value theorem. For

$$
\eta_{1}(t)-B \in\left[\int_{a}^{t} r_{2}(s) \phi_{\alpha_{2}}^{-1}\left[C+I_{2}(s)\right] \mathrm{d} s, \int_{a}^{t} r_{2}(s) \phi_{\alpha_{2}}^{-1}\left[C+I_{1}(s)\right] \mathrm{d} s\right]
$$

and

$$
\eta_{2}(t) \in\left[C+I_{2}(s), C+I_{1}(s)\right]
$$

we obtain

$$
y_{01}(t)-y_{02}(t)=\frac{1}{\beta} \int_{a}^{t}\left|\eta_{1}(s)\right|^{\frac{1-\alpha_{1}}{\alpha_{1}}} r_{1}(s) \int_{a}^{s}\left|\eta_{2}(u)\right|^{\frac{1-\alpha_{2}}{\alpha_{2}}} r_{2}(u)\left(I_{1}(u)-I_{2}(u)\right) \mathrm{d} u \mathrm{~d} s .
$$

Let $t \geq a$. Then for $t \rightarrow a$ we have $B+\int_{a}^{t} r_{2}(s) \phi_{\alpha_{2}}^{-1}\left[C+I_{i}(s)\right] \mathrm{d} s \rightarrow B \neq 0$ and $C+I_{i}(s) \rightarrow C \neq 0, \quad i=1,2$. Therefore there exist $\epsilon_{1}>0, \epsilon_{2}>0$ s.t.

$$
\frac{1}{2}|B| \leq\left|\eta_{1}(t)\right| \leq \frac{3}{2}|B| \quad \text { for } \quad t \in\left[a, a+\epsilon_{1}\right]
$$

and

$$
\frac{1}{2}|C| \leq\left|\eta_{2}(t)\right| \leq \frac{3}{2}|C| \quad \text { for } \quad t \in\left[a, a+\epsilon_{2}\right]
$$

Denote by

$$
M(B)= \begin{cases}\frac{1}{2}|B|, & \text { if } \quad \alpha_{1}>1 \\ \frac{3}{2}|B|, & \text { if } \quad \alpha_{1} \leq 1\end{cases}
$$




\section{JOZEF KISEL'ÁK}

and

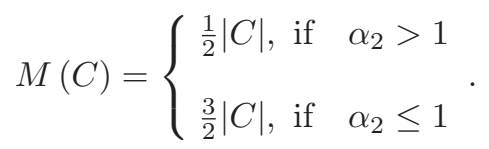

Then using the notation $L=M(B)^{\frac{1-\alpha_{1}}{\alpha_{1}}} M(C)^{\frac{1-\alpha_{2}}{\alpha_{2}}} / \beta$ we have

$$
\begin{gathered}
\left|y_{01}(t)-y_{02}(t)\right| \leq L \int_{a}^{t} r_{1}(s) \int_{a}^{s} r_{2}(u)\left|I_{1}(u)-I_{2}(u)\right| \mathrm{d} u \mathrm{~d} s= \\
=L \int_{a}^{t}\left(\int_{s}^{t} r_{1}(u) \mathrm{d} u\right) r_{2}(s)\left|I_{1}(s)-I_{2}(s)\right| \mathrm{d} s
\end{gathered}
$$

for $t \in\left[a, a+\min \left\{\epsilon_{1}, \epsilon_{2}\right\}\right]$. Furthermore, define the continuous function $z_{i}(t)$ by

$$
z_{i}(t)=\left\{\begin{array}{c}
\frac{y_{0 i}(t)}{\int_{a}^{t} r_{1}(s) \mathrm{d} s}, t \neq a \\
\phi_{\alpha_{1}}^{-1}[B], \quad t=a
\end{array},\right.
$$

for $i=1,2$. Then we see from previous estimate that

$$
\begin{gathered}
\left|z_{1}(t)-z_{2}(t)\right| \leq L \int_{a}^{t} r_{2}(s) \int_{a}^{s}\left(\int_{a}^{u} r_{1}(v) \mathrm{d} v\right)^{\beta}|q(u)|\left|\phi_{\beta}\left[z_{1}(u)\right]-\phi_{\beta}\left[z_{2}(u)\right]\right| \mathrm{d} u \mathrm{~d} s \\
=L \int_{a}^{t}\left(\int_{s}^{t} r_{2}(u) \mathrm{d} u\right)\left(\int_{a}^{s} r_{1}(v) \mathrm{d} v\right)^{\beta}|q(s)|\left|\phi_{\beta}\left[z_{1}(s)\right]-\phi_{\beta}\left[z_{2}(s)\right]\right| \mathrm{d} s .
\end{gathered}
$$

Again we use mean value theorem. A constant $\epsilon^{\prime}<\min \left\{\epsilon_{1}, \epsilon_{2}\right\}$ can be chosen so that

$$
\left|\phi_{\beta}\left[z_{1}(s)\right]-\phi_{\beta}\left[z_{2}(s)\right]\right| \leq \beta\left|\eta_{3}(t)\right|^{\beta-1}\left|z_{1}(t)-z_{2}(t)\right|,
$$

$\eta_{3}(t)$ lying between $z_{1}(t)$ and $z_{2}(t)$. Since $\beta<1$ we have estimate $\left|\eta_{3}(t)\right| \leq \frac{3}{2}|B|^{\frac{1}{\alpha_{1}}}$. Therefore for $K=\left(\frac{3}{2}\right)^{\beta-1} \beta|B|^{\frac{\beta-1}{\alpha_{1}}} L$ we finally achieve estimation

$$
\left|z_{1}(t)-z_{2}(t)\right| \leq K \int_{a}^{t} g(s, t)\left|z_{1}(s)-z_{2}(s)\right| \mathrm{d} s
$$

for $t \in\left[a, a+\epsilon^{\prime}\right]$, where $g(s, t)=|q(s)|\left(\int_{s}^{t} r_{2}(u) \mathrm{d} u\right)\left(\int_{a}^{s} r_{1}(v) \mathrm{d} v\right)^{\beta}$. We conclude that $z_{1}=z_{2}$ on $t \in\left[a, a+\epsilon^{\prime}\right]$, which obviously implies that $y_{01}=y_{02}$ on $t \in\left[a, a+\epsilon^{\prime}\right]$. For a small left neighborhood of $a$ we can proceed similarly. This completes the proof.

Lemma 3.4. Suppose that $A \neq 0, B=0, C=0$ and $\alpha_{1}>1, \alpha_{2}>1$ and $q(a) \neq 0$. Then the IVP $(2)$ with $t_{0}=$ a has a unique solution in a right neighborhood of the point a.

Proof. Let $y_{01}$ and $y_{02}$ be two local solutions of (2) with assumptions above. Now, let $t \in$ $\operatorname{dom}\left(y_{01}\right) \cap$ 
$\operatorname{dom}\left(y_{02}\right)$ and integrate (8) with $y=y_{0 i}$ three times from $a$ to $t$. If we denote as $I_{i}(t)=\int_{a}^{t} q(s) \phi_{\beta}\left[y_{0 i}(s)\right] \mathrm{d} s$, we have

$$
y_{0 i}(t)=A+\int_{a}^{t} r_{1}(s) \phi_{\alpha_{1}}^{-1}\left[\int_{a}^{s} r_{2}(u) \phi_{\alpha_{2}}^{-1}\left[I_{i}(u)\right] \mathrm{d} u\right] \mathrm{d} s, \quad i=1,2 .
$$

Define the continuous functions on $[a, a+\delta], \delta>0$ as

$$
J_{i}(t)=\left\{\begin{array}{c}
\frac{I_{i}(t)}{t-a}, \quad t \neq a \\
q(a) \phi_{\beta}[A], \quad t=a
\end{array}\right.
$$

and

$$
K_{i}(t)=\left\{\begin{array}{cc}
\frac{\frac{1+\alpha_{2}}{\alpha_{2}} \int_{a}^{t} r_{2}(s)(s-a)^{\frac{1}{\alpha_{2}}} \phi_{\alpha_{2}}^{-1}\left[J_{i}(s)\right] \mathrm{d} s}{(t-a)^{\frac{1}{\alpha_{2}}+1}}, & t \neq a \\
r_{2}(a) \phi_{\alpha_{2}}^{-1}[q(a)] \phi_{\alpha_{1}}[A], & t=a
\end{array} .\right.
$$

But then we obtain

$$
\left|y_{01}(t)-y_{02}(t)\right| \leq c \int_{a}^{t} \tilde{r}_{1}(s)\left|\phi_{\alpha_{1}}^{-1}\left[K_{1}(s)\right]-\phi_{\alpha_{1}}^{-1}\left[K_{2}(s)\right]\right| \mathrm{d} s
$$

where $\tilde{r}_{1}(s)=r_{1}(s)(s-a)^{\frac{1}{\beta}+\frac{1}{\alpha_{1}}}, c=\left(\frac{\alpha_{2}}{\alpha_{2}+1}\right)^{\frac{1}{\alpha_{1}}}$. Now we are able to use mean value theorem and one can proceed further as in the proof of lemma 3.3.

Lemma 3.5. Suppose that $A=0, B=0, C \neq 0$ and $\alpha_{1}>1, \quad \beta<1$. Then the $\operatorname{IVP}(2)$ with $t_{0}=a$ has a unique solution in a right neighborhood of the point $a$.

Proof. Let $y_{01}$ and $y_{02}$ be two local solutions of (2) with assumptions above. Now, let $t \in$ $\operatorname{dom}\left(y_{01}\right) \cap$

$\operatorname{dom}\left(y_{02}\right)$ and integrate (8) with $y=y_{0 i}$ three times from $a$ to $t$. If we denote as $I_{i}(t)=\int_{a}^{t} q(s) \phi_{\beta}\left[y_{0 i}(s)\right] \mathrm{d} s$, we have

$$
y_{0 i}(t)=\int_{a}^{t} r_{1}(s) \phi_{\alpha_{1}}^{-1}\left[\int_{a}^{s} r_{2}(u) \phi_{\alpha_{2}}^{-1}\left[C+I_{i}(u)\right] \mathrm{d} u\right] \mathrm{d} s, \quad i=1,2 .
$$

Now set the continuous function on $[a, a+\delta], \delta>0$

$$
K_{i}(t)=\left\{\begin{array}{cc}
\frac{\int_{a}^{t} r_{2}(s) \phi_{\alpha_{2}}^{-1}\left[C+I_{i}(s)\right] \mathrm{d} s}{(t-a)}, & t \neq a \\
r_{2}(a) \phi_{\alpha_{2}}^{-1}[C], & t=a
\end{array} .\right.
$$

But then we obtain

$$
\left|y_{01}(t)-y_{02}(t)\right| \leq \int_{a}^{t} \tilde{r}_{1}(s)\left|\phi_{\alpha_{1}}^{-1}\left[K_{1}(s)\right]-\phi_{\alpha_{1}}^{-1}\left[K_{2}(s)\right]\right| \mathrm{d} s,
$$

where $\tilde{r}_{1}(s)=r_{1}(s)(s-a)^{\frac{1}{\alpha_{1}}}$. One can proceed further as in the proof of lemma 3.3 . 


\section{JOZEF KISEL'ÁK}

Lemma 3.6. Suppose that $A=0, B \neq 0, C=0$ and $\alpha_{2}>1, \beta<1$ and $q(a) \neq 0$. Then the IVP (2) with $t_{0}=a$ has a unique solution in a right neighborhood of the point a.

Proof. Let $y_{01}$ and $y_{02}$ be two local solutions of (2) with assumptions above. Now, let $t \in$ $\operatorname{dom}\left(y_{01}\right) \cap$

$\operatorname{dom}\left(y_{02}\right)$ and integrate (8) with $y=y_{0 i}$ three times from $a$ to $t$. If we denote as $I_{i}(t)=\int_{a}^{t} q(s) \phi_{\beta}\left[y_{0 i}(s)\right] \mathrm{d} s$, we have

$$
y_{0 i}(t)=\int_{a}^{t} r_{1}(s) \phi_{\alpha_{1}}^{-1}\left[B+\int_{a}^{s} r_{2}(u) \phi_{\alpha_{2}}^{-1}\left[I_{i}(u)\right] \mathrm{d} u\right] \mathrm{d} s, \quad i=1,2 .
$$

Define the continuous function on $[a, a+\delta], \delta>0$

$$
J_{i}(t)=\left\{\begin{array}{c}
\frac{I_{i}(t)}{t-a}, t \neq a \\
0, \quad t=a
\end{array} .\right.
$$

But then we obtain

$$
\left|y_{01}(t)-y_{02}(t)\right| \leq \int_{a}^{t} r_{1}(s)\left|\phi_{\alpha_{1}}^{-1}\left[K_{1}(s)\right]-\phi_{\alpha_{1}}^{-1}\left[K_{2}(s)\right]\right| \mathrm{d} s,
$$

where $K_{i}(s)=B+\int_{a}^{s} r_{2}(s)(s-a)^{\frac{1}{\alpha_{2}}} \phi_{\alpha_{2}}^{-1}\left[J_{i}(u)\right] \mathrm{d} u$. One can proceed further as in the proof of lemma 3.3 .

Lemma 3.7. The IVP (2) with $A=B=C=0$ possess only trivial solution $y(t)=0$.

Remark 3.8. The statement follows directly from the proof of the global existence, since initial conditions imply $M=0$.

We can prove similarly all assertions in the case of left neighborhood of the point $t_{0}$. Finally we can propose the main statement of the section which is summarized in the following theorem.

Theorem 3.9. Suppose that $\beta=\alpha_{1} \alpha_{2}$ and $q(a) \neq 0$. Given $a \in I$ and $A, B, C \in$ $\mathbb{R}$, there exists a unique solution in a neighborhood of the point a of the IVP (2).

But this is not true generally for "non constrained" $\beta$ as following examples for the case $\beta<\alpha_{1} \alpha_{2}$ show.

Example 3.10. Consider the IVP

$$
\begin{array}{ll}
x_{0}^{\prime}=\left|x_{1}\right|^{\frac{1-\alpha_{1}}{\alpha_{1}}} x_{1}, & x_{0}(0)=0 \\
x_{1}^{\prime}=x_{2}, & x_{1}(0)=0 \\
x_{2}^{\prime}=\left|x_{0}\right|^{\frac{1}{3}} x_{0}, & x_{2}(0)=0
\end{array}
$$

Moreover, we suppose that $\alpha_{1}>\frac{4}{3}=\beta$. But, except the trivial solution, there exist another one solution of the problem (11), namely

$$
\left\{x_{0}(t)=k t^{c}, \quad x_{1}(t)=(c k)^{\alpha_{1}} t^{\alpha_{1}(c-1)}, \quad x_{2}(t)=\alpha_{1}(c k)^{\alpha_{1}}(c-1) t^{\alpha_{1}(c-1)-1}\right\},
$$


where $c=\frac{3\left(\alpha_{1}+2\right)}{3 \alpha_{1}-4}, \frac{1}{k}=\frac{10 \alpha_{1}\left(7 \alpha_{1}+4\right)\left(\alpha_{1}+2\right)^{\alpha_{1}}}{\left(9 \alpha_{1}^{2}-24 \alpha_{1}+16\right)\left(3 \alpha_{1}-4\right)^{\alpha_{1}}}$. Notice that $\alpha_{1}=\frac{4}{3}$ (specific boundary case) is the root of the expressions contained in $c, k$ and this does not happen by coincidence.

Example 3.11. Consider the IVP

$$
\begin{array}{ll}
x_{0}^{\prime}=x_{1}, & x_{0}(0)=0 \\
x_{1}^{\prime}=\left|x_{2}\right|^{\frac{1-b}{b}} x_{2}, & x_{1}(0)=0 \\
x_{2}^{\prime}=\left|x_{0}\right|^{-\frac{2+b}{2(1+b)}} x_{0}, & x_{2}(0)=0
\end{array}
$$

Certainly (for $b>0$ ) we have $\alpha_{2}=b>\frac{b}{2(1+b)}=\beta$ and $0<\beta \leq \frac{1}{2}$. But, there exist infinitely many solutions of the problem (12). Denote by $k>0$ a solution of

$$
\frac{2 b^{3}\left(2+3 b+b^{2}\right)^{b}\left(2^{1+b} b^{-2 b-3}+3 b^{-2 b-2} 2^{b}+b^{-2 b-1} 2^{b}\right)}{(1+b)(2+b)}=k^{b-\frac{b}{2(1+b)}},
$$

then

$$
\chi_{c}(t)\left\{\frac{(t-c)^{\frac{1}{\beta}}}{k}, \frac{(t-c)^{\frac{1}{\beta}-1}}{\beta k}, \frac{(1-\beta)^{\beta}}{(\beta k)^{\beta}}(t-c)^{1-2 \beta}\right\}
$$

where

$$
\chi_{c}(t)=\left\{\begin{array}{c}
0,0 \leq t \leq c, \\
1, \quad t \geq c
\end{array},\right.
$$

is the solution of the problem (12) for all nonnegative $c$.

There arises the question whether it is true for the case of $\beta>\alpha_{1} \alpha_{2}$. We expect that continuity conditions on coefficients $r_{1}, r_{2}, q$ are not sufficient for uniqueness as results for second-order equations suggest. In [Erbe and Liang 1991] author shows that for second-order quasi-linear differential equation

$$
\left(r(t) \phi_{\alpha}\left[y^{\prime}\right]\right)^{\prime}+q(t) \phi_{\beta}[y]=0,
$$

the quotient $\frac{r}{q}$ must be of locally bounded variation. In [Kitano and Kusano 1995], for equation (13) with $r(t)=1$, stronger condition is offered, namely $q \in C^{1}(I)$. Nevertheless, we left it here as an open problem. 


\section{JOZEF KISEL'ÁK}

\section{REFERENCES}

Coffman, C. And Ullrich, D. 1967. On the continuation of solutions of a certain non-linear differential equation. Monatsh. Math. 71, 385-392.

Erbe, L. H. ANd Liang, Z. 1991. Continuation and uniqueness for generalized Emden-Fowler systems. J. Aust. Math. Soc., Ser. B 33, 1, 85-93.

Flajolet, P., Dumas, P., And Puyhaubert, V. 2006. Some exactly solvable models of urn process theory. In Proceedings of Fourth Colloquium on Mathematics and Computer Science, P. Chassaing, Ed. Discrete Mathematics and Theoretical Computer Science, vol. AG. 59-118.

Kitano, M. And Kusano, T. 1995. On a class of second order quasilinear ordinary differential equations. Hiroshima Math. J. 25, 321-355.

Mirzov, J. 1976. On some analogs of Sturm's and Kneser's theorems for nonlinear systems. J. Math. Anal. Appl. 53, 418-425.

Ollagnier, J. M., Nowicki, A., And Strelcyn, J.-M. 1995. On the nonexistence of constants of derivations: the proof of a theorem of Jouanolou and its development. Bull. Sci. Math. 119, 3, $195-233$.

Reichel, W. And Walter, W. 1997. Radial solutions of equations and inequalities involving the p-Laplacian. J. Inequal. Appl. 1, 1, 47-71.

Jozef Kisel'ák,

Institute of Mathematics, Faculty of Science

P.J. Šafárik University in Košice

Jesenná 5, 04001 Košice

Slovak Republic,

http://umv.science.upjs.sk/,

email: jozef.kiselak@upjs.sk

Received November 2013 